\title{
Actualidad institucional y económica de España en el marco de la Unión Europea (Junio 2021)
}

\author{
Beatriz Iñarritu \\ Profesora de la «Deusto Business School», Universidad de Deusto
}

doi: http://dx.doi.org/10.18543/ced-65-2021pp229-248

Sumario: I. Introducción.-II. El estado de la Integración.III. Cuestiones generales de la actualidad económica

\section{Introducción}

A lo largo del primer semestre de 2021 la Unión ha continuado la implementación de los programas de estímulos fiscales del paquete «Next Generation EU» y estímulos monetarios del Banco Central Europeo, y hemos conocido la aprobación de los diferentes Planes Nacionales de Recuperación, Transformación y Resiliencia, incluido el español.

Por otra parte, también fue un tema relevante del semestre la inauguración de la «Conferencia sobre el Futuro de Europa», con la que se iniciaba un novedoso proceso de reflexión ciudadana y política que planteará, previsiblemente, cambios importantes en el funcionamiento y en las prioridades de la Unión del futuro.

La reactivación del Pilar de los Derechos Sociales, la reforma de la Política Agrícola Común, el futuro del Pacto de Estabilidad y Crecimiento, la transparencia fiscal de las grandes corporaciones y las diferentes investigaciones llevadas a cabo por la Dirección General de la Competencia de la Comisión Europea fueron, asimismo, asuntos destacados en este primer semestre de 2021.

During this first half of 2021 the Union has continued to implement the fiscal stimulus from the "Next Generation EU" pack and those monetary stimulus on the part of the European Central Bank, and therefore we have known the approval of different National Plans of Recovery, Transformation and Resilience, the Spanish one included. 
On the other hand, it was also an outstanding point in the semester the launch of the "Conference on the Future of Europe" which initiated an innovative process of public and political reflexion that might propose important changes in the EU's functioning and priorities.

Other remarkable issues in this first semester of 2021 were the activation of the Social Agenda, the reform of the Common Agricultural Policy, the future Growth and Stability Pact, the tax transparency of big companies and the different investigations carried out by the Directorate General for Competition of the European Commission.

\section{El estado de la integración}

\section{Los Fondos de la Recuperación: aprobación del Plan Nacional de} Recuperación español y emisión de Deuda conjunta

La presidenta de la Comisión Europea, Ursula von der Leyen, se desplazó a Madrid el 16 de Junio para comunicar en persona al presidente del gobierno español, Pedro Sánchez, la aprobación del Plan de Recuperación de España.

España tendrá acceso a 140.000 millones de euros procedentes de los fondos «Next Generation EU», de los que 69.500 serán ayudas directas, y que irá recibiendo cada año hasta 2026. El gobierno español deberá demostrar la ejecución de una serie de hitos y objetivos para asegurarse el cobro de los 69.500 millones y, si finalmente decide solicitarlos, de otros 70.500 millones de euros en préstamos en condiciones ventajosas.

Por tanto, España deberá ir certificando el cumplimiento de una lista de 212 medidas contempladas en el Plan, 102 reformas y 110 inversiones.

«El plan pone un enfoque positivo en la creación de empleo y en la próxima generación, con medidas para abordar el desempleo juvenil, mejorar la provisión de habilidades relevantes para el mercado laboral y mejorar el entorno empresarial y la administración pública», destacó el vicepresidente económico de la Comisión, Valdis Dombrovskis.

En todo caso, junto a las reformas también son relevantes las inversiones, ya que el Plan aprobado por la Comisión prevé movilizar un volumen de recursos sin precedentes para acometer 110 proyectos de inversión, centrados fundamentalmente en los ámbitos de la transición ecológica y la digitalización.

Von der Leyen destacó, en este sentido, que el $40 \%$ de las inversiones españolas girarán en torno a la transición ecológica y un $28 \%$ se centrarán en la transformación digital, un porcentaje superior al $20 \%$ que solicitaba la Comisión. 
Los diez proyectos de inversión clave recibirán 50.000 millones de euros para su despliegue. Los más importantes estarán relacionados con la movilidad y el coche eléctrico, que recibirán unos 13.200 millones de euros, mientras que la renovación de edificios para mejorar su eficiencia energética recibirá 6.800 millones. El Plan español también destinará 3.100 millones para el despliegue e integración de energías renovables, e impulsará la «transición verde» del sector turístico con 3.400 millones de euros.

En el apartado digital, el plan reserva 4.300 millones para la modernización de las administraciones públicas y otros 4.000 millones estarán destinados a ayudar a las pymes. Además, se invertirán 4.000 millones en el despliegue del 5G y se apostará por la formación con una inversión de 3.600 millones para un plan nacional de competencias digitales.

La recomendación de la Comisión deberá pasar al Consejo de la UE, que dispondrá de hasta cuatro semanas para evaluar y respaldar definitivamente el plan, una decisión que podría ser adoptada en la reunión de ministros de Finanzas de la UE (Ecofin) que se celebrará a mediados de julio, desbloqueando así la entrega de los primeros fondos de prefinanciación que llegarían al gobierno español ese mismo mes.

España espera un desembolso anticipado de 9.000 millones de euros en concepto de prefinanciación del Plan antes de Agosto de 2021 y otros 10.000 en Diciembre a cuenta de las inversiones y reformas ya ejecutadas.

A partir de 2022, se efectuarán otros ocho desembolsos semestrales hasta 2026, que estarán necesariamente sujetos al control del cumplimiento de las reformas e inversiones marcadas en el Plan, y de los hitos y objetivos correspondientes. 


\section{Plan de recuperación, transformación y resiliencia Distribución de la inversión}

\begin{tabular}{|c|c|c|c|}
\hline \multicolumn{2}{|r|}{ Tabla 10: Distribución de la inversión entre las diferentes palancas y componentes } & ME & $\%$ \\
\hline \multirow{4}{*}{ 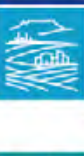 } & I. Agenda urbana y rural. lucha contra la despoblación y deserrollo de la agricultura & 14.407 & $20.7 \%$ \\
\hline & 1. Plan de choque de movilidad sostenible, segura y conectada en entornos urbanos y metropolitanos & 6.536 & $9,6 \%$ \\
\hline & 2. Plan de rehabilitacion de vivienda y regeneracion urbana & 6.820 & $9.8 \%$ \\
\hline & 3. Transformación ambiental y digital del sistema agroalimentario y pesquero & 1.051 & $1.5 \%$ \\
\hline \multirow{4}{*}{$\sum_{2}^{-1}$} & II. Infraestructuras y ecosistemas resilientes & 10.400 & $15.0 \%$ \\
\hline & 4. Conservación y restauración de ecosistemas y su biodiversidad & 1.642 & $2,6 \%$ \\
\hline & 5. Preservación del espacio litoral y los recursos hídricos & 2.091 & $3,0 \%$ \\
\hline & 6. Movilidad sostenible, segura y conectada & 6.667 & $9,6 \%$ \\
\hline \multirow{5}{*}{$\frac{1+2}{4 x}$} & III. Transición energética justa e inclusiva & 6.385 & $9.2 \%$ \\
\hline & 7. Despliegue e integración de energias renovables & 3.165 & $4,6 \%$ \\
\hline & 8. Infraestructuras eléctricas, promoción de redes inteligentes y despliegue de la flexibilidad y el almacenamiento & 1.365 & $2,0 \%$ \\
\hline & 9. Hoja de ruta del hidrógeno renovable y su integraciön sectorial & 1.555 & $2,2 \%$ \\
\hline & 10. Estrategla de Transición Justa & 300 & $0.6 \%$ \\
\hline \multirow{2}{*}{ बं० } & IV. Una Administración para el siglo XXI & 6.315 & $6,2 \%$ \\
\hline & 11. Modernización de las Administraciones públicas & 4.315 & $6.2 \%$ \\
\hline \multirow{5}{*}{$\begin{array}{l}3 \% \\
2 \sqrt{\circ}\end{array}$} & $\begin{array}{l}\text { V. Modernización y digitalización del tejido industrial y de la pyme. recuperación del turismo e } \\
\text { impulso a una Espan̂a nación emprandedara }\end{array}$ & 16.075 & $23,1 \%$ \\
\hline & 12. Potitica Industrial España 2030 & 3.782 & $5.6 \%$ \\
\hline & 13. Impulso a la pyme & 4.896 & $7,0 \%$ \\
\hline & 16. Plan de modernización y competitividad del sector turistico & 3.600 & $6,9 \%$ \\
\hline & 15. Conectividad Digital, Imputso de la clberseguridad y despliegue del 56 & 3.999 & $5.8 \%$ \\
\hline \multirow{4}{*}{ E } & VI. Pacto por la ciencia y la innovación. Refuerzo a las capacidades del Sistema Nacional de Salud & 4.949 & 7,12 \\
\hline & 16. Estrategia Nacional de Inteligencia Artificial & 500 & $0,7 \%$ \\
\hline & 17. Reforma institucional y fortalecimiento de las capacidades del sistema nacional de ciencia, tecnología e innovación & 3.380 & $4.9 \%$ \\
\hline & 18. Renowacion y ampliación de las capacidades dol Sistema Nacional de Satud & 1.069 & $1.5 \%$ \\
\hline \multirow{4}{*}{ 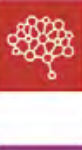 } & VII. Educación y conocimiento. formación continua y desarrollo de capacidades & 7.317 & $10.5 \%$ \\
\hline & 19. Plan Nacional de Competencias Digitales (digital skills) & 3.593 & $5,2 \%$ \\
\hline & 20. Plan estratégico de impulso de la Formacion Profesional & 2.076 & $3.0 \%$ \\
\hline & 21. Modernización y digitalización del sistema educativo, incluida la educación temprana de 0 a 3 anos & 1.648 & $2,6 \%$ \\
\hline \multirow{3}{*}{ (1) } & VIII. Nueva economia de los cuidados y politicas de empleo & 4.855 & $7.0 \%$ \\
\hline & 22. Plan de choque para la economia de los cuidados y refuerzo de las politicas de inclusión & 2.692 & $3.6 \%$ \\
\hline & 23. Nuevas politicas püblicas para un mercado de trabajo dinámico, resiliente e inclusivo & 2.363 & $3,6 \%$ \\
\hline \multirow{4}{*}{$\$ 8$} & IX. Impulso de la industria de la cultura y el deporte & 825 & 1.27 \\
\hline & 24. Revalorización de la industria cultural & 325 & $0.5 \%$ \\
\hline & 25. España hub audiovisual de Europa (Spain AVS Hub) & 200 & $0,3 \%$ \\
\hline & 26. Plan de fonento del sector del deporte & 300 & $0,6 \%$ \\
\hline \multirow{6}{*}{ 政IIII } & X. Modernización del sistema fiscal para un crecimiento inclusivo y sostenible & - & - \\
\hline & 27. Medidas y actuaciones de prevencion y lucha contra el fraude fiscal & - & - \\
\hline & 28. Adaptación del sistema impositivo a la realidad del siglo XXI & - & - \\
\hline & 29. Mejora de la eflicacia del gasto público & - & - \\
\hline & 30. Sostenibilidad a largo plazo del sistema público de pensiones en el marco del Pacto de Toledo & - & - \\
\hline & Total & 69.528 & $100 \%$ \\
\hline
\end{tabular}


Plan de recuperación, transformación y resiliencia

Principales reformas y programas tractores de inversión

\begin{tabular}{|c|c|}
\hline RELA 2. Les 20 propranes tractares do inversión & MC 2021-2] \\
\hline 1. Estrateja de Mevilidut Sosten be, Segua y tooectato & 1230 \\
\hline 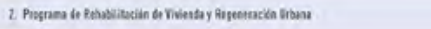 & 6500 \\
\hline 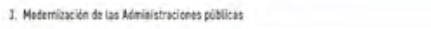 & ans \\
\hline 6. Pla de Olacalinacias de Pran & atse \\
\hline 5. Hoia te Rata del So & $3 m$ \\
\hline 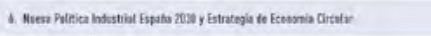 & se \\
\hline 7. Plan Katienal de Conpettecias Degitales & $25 n$ \\
\hline 1. Mostenilak ke y Coapresivitad tel Secter Tritike & 3600 \\
\hline 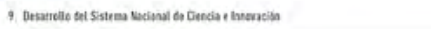 & 3180 \\
\hline 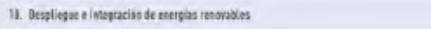 & 3165 \\
\hline 11. Numa Ecaneñáa de les Codtates & $2 \sin 2$ \\
\hline 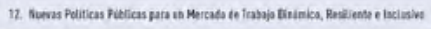 & 2162 \\
\hline 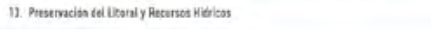 & $2 m$ \\
\hline 16. Pan Eatratioca de Farnacion Prolesicnal & $2 m$ \\
\hline 15. Moterisacian y Diviliencias del Satuma fexcative & tus \\
\hline 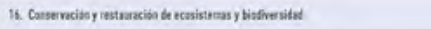 & 160 \\
\hline 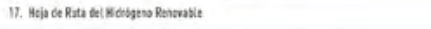 & 155 \\
\hline 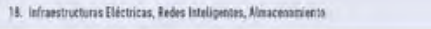 & 1145 \\
\hline 19. Renercibn yoterniacian del Sitana Sentaris & tuss \\
\hline 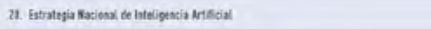 & 10 \\
\hline
\end{tabular}

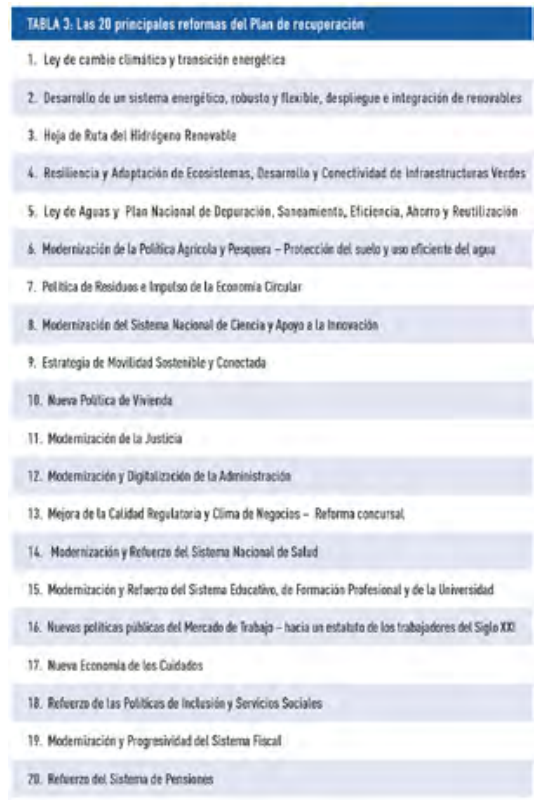

Fuente: Gobierno de España.

https://www.lamoncloa.gob.es/temas/fondos-recuperacion/Paginas/index.aspx https://www.lamoncloa.gob.es/temas/fondos-recuperacion/Documents/30042021-Plan_Recuperacion_\%20Transformacion_\%20Resiliencia.pdf

Para financiar estos Fondos europeos de Recuperación, conocidos como «Next Generation EU», y, por tanto, los diferentes Planes nacionales, Bruselas anunció a comienzos de Junio el lanzamiento de la primera emisión de deuda conjunta.

Desde este mismo mes de Junio y hasta finales de 2026, la Comisión Europea prevé emitir deuda por valor de unos 150.000 millones de euros al año, haciendo valer sus más altas calificaciones crediticias conseguidas ante los inversores y previendo un plazo de amortización de la deuda que se extenderá hasta 2058.

Los analistas y expertos financieros no han dudado en destacar la relevancia de esta mutualización masiva de deuda, y han señalado que, incluso, podría ser el embrión de un futuro Tesoro europeo. 


\title{
NextGenerationEU - a game changer in EU capital markets
}

\begin{abstract}
NextGenerationEU is a temporary recovery instrument which can raise up to 6750 billion in 2018 prices or some $\mathbf{6 8 0 0}$ billion in current prices through bond issuance. It is at the heart of the European Union (EU) response to the coronavirus crisis and aims to support the economic recovery and build a greener, more digital and more resilient future.
\end{abstract}

To finance NextGenerationEU, the European Commission, on behalf of the EU, will borrow on the capital markets. The borrowing will be undertaken between mid-2021 and 2026. All borrowing will be repaid by 2058 .

This would translate into borrowing volumes of on average roughly $€ 150$ billion per year between mid-2021 and 2026, which will make the EU one of the largest issuers in euro. By using diverse funding instruments and funding techniques, the Commission will expand

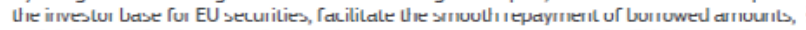
and deliver all funds as required on the most advantageous terms for EU Member States and citizens.

Given the volumes, frequency and complexity of the borrowing operations ahead, the Commission will follow the best practices used by sovereign issuers, and implement a diversified funding strategy.

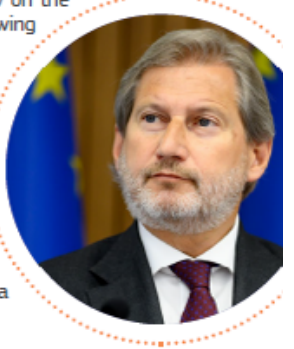

Fuente: Comisión Europea.

https://ec.europa.eu/info/sites/default/files/about_the_european_commission/eu_budget/factsheet_1_funding_strategy_20.04.pdf

https://ec.europa.eu/info/strategy/eu-budget/eu-borrower-investor-relations_ es\#nextgenerationeu

En total, la Comisión prevé demandar a los mercados hasta 800.000 millones de euros entre 2021 y 2026, convirtiéndose así en uno de los mayores actores mundiales del mercado de deuda.

\section{La Conferencia sobre el futuro de la UE}

El pasado 9 de Mayo, día de Europa, la Unión Europea puso en marcha, en la sede del Parlamento Europeo de Estrasburgo, un novedoso ejercicio de participación ciudadana y reflexión política sobre su futuro bautizado como la «Conferencia sobre el Futuro de Europa», CFE, que deberá prolongarse durante doce meses, hasta el 9 de Mayo de 2022.

De hecho, el coronavirus había obligado a retrasar el inicio de la Conferencia, ya que inicialmente estaba prevista su inauguración para el 9 de mayo de 2020, coincidiendo con el 70 aniversario de la Declaración de Schuman. Finalmente, se ha puesto en marcha en el 71 aniversario a pesar de que varias capitales eran partidarias de cancelarla definitivamente y a pesar también del enfrentamiento mostrado entre las tres instituciones involucradas, Parlamento Europeo, Comisión Europea y Consejo de la UE, por asumir el liderazgo y la gestión del nuevo foro, y cuya solución fue alcanzada con una presidencia tripartita y paritaria. 
La Conferencia estará liderada, en efecto, por la triple co-presidencia del eurodiputado Guy Verhofstadt, de la vicepresidenta de la Comisión Dubravka Suica y de un representante de la presidencia rotatoria del Consejo de la UE, representación que, durante el primer semestre de 2021, ha sido asumida por la secretaria de Estado portuguesa, Ana Paula Zacarias, y quien será sustituida por representantes de los gobiernos esloveno y francés, puesto que estos dos países asumirán las dos próximas presidencias rotatorias del segundo semestre de 2021 y del primero de 2022.

Un Comité Ejecutivo, compuesto por nueve miembros, tres por cada una de las instituciones implicadas en la Conferencia (Comisión, Parlamento y Consejo) y cuatro observadores con voz pero sin voto, organizará la Conferencia (las sesiones Plenarias, los Paneles Ciudadanos, la Plataforma Digital Multilingüe y sus eventos), la supervisará a medida que avance y monitorizará las aportaciones de los ciudadanos. El Comité Ejecutivo asistirá e informará periódicamente a la Presidencia Conjunta.

Por otra parte, y dado que el objetivo de la Conferencia es conocer las opiniones de la ciudadanía para intentar después traducirlas en posibles reformas de la UE, se constituirán cuatro paneles con 200 ciudadanos cada uno, que deberán ser seleccionados respetando la diversidad de la UE en términos de origen geográfico, edad, situación socioeconómica y nivel educativo, según las normas pactadas por las tres instituciones europeas. Se garantizará, en este sentido, la presencia de al menos un hombre y una mujer de cada uno de los 27 países de la UE, y que al menos un tercio de los miembros tengan una edad comprendida entre los 16 y los 25 años.

Además de estos paneles, la Conferencia ha activado una plataforma digital y multilingüe para que cualquier ciudadano que se registre tenga la oportunidad de participar en los debates en marcha o, incluso, organizar su propio debate o acto de deliberación.

Las propuestas se trasladarán al Plenario de la Conferencia para ser debatidas. Este pleno estará compuesto por 433 personas, 108 eurodiputados, 54 representantes del Consejo de la UE (dos por Estado miembro) y 3 de la Comisión Europea; y también serán miembros del Plenario 108 diputados nacionales, 108 ciudadanos (80 representantes de los paneles europeos, 27 representantes de los paneles o actos celebrados a nivel nacional y el presidente del Foro europeo juvenil), 18 miembros del Comité de las Regiones, 18 miembros del Comité Económico y Social, 8 representantes de los agentes sociales y otros 8 de la sociedad civil.

El Alto Representante de la UE para la Política Exterior podrá participar cuando se discuta sobre el papel internacional de la UE, y otros actores relevantes podrán ser invitados puntualmente.

Las conclusiones del Plenario pasarán al Comité, quien se encargará de presentar el informe final a la presidencia conjunta. Las tres instituciones 
deberán, entonces, decidir cómo implementar, en su caso, las propuestas recibidas, en el marco de las competencias que tengan asignadas en los Tratados. Las propuestas podrían dar lugar, de esta forma, a una eventual y profunda transformación de la UE.

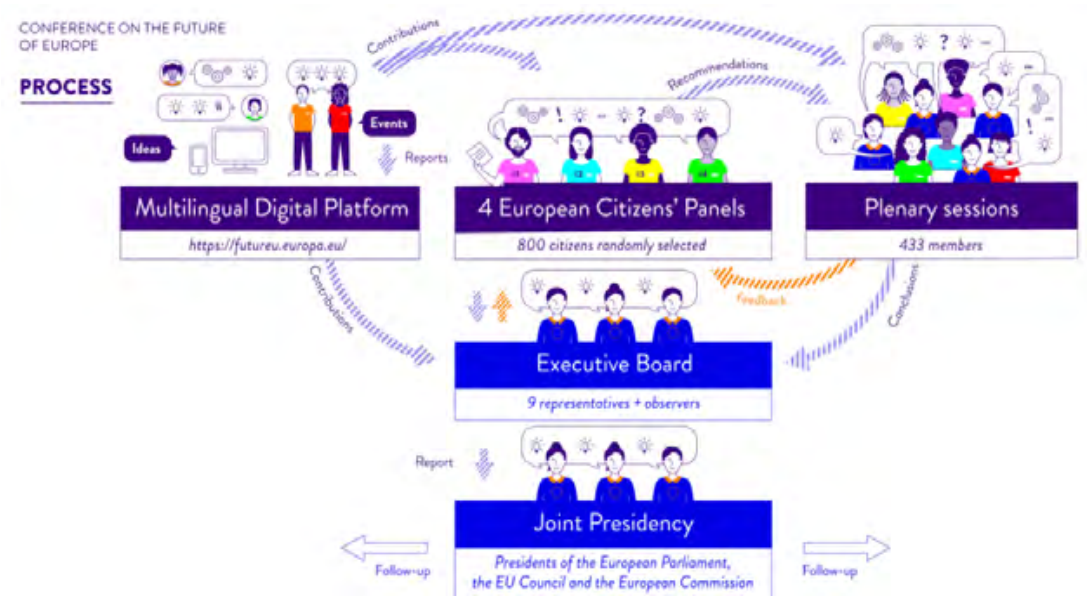

Fuente: Conferencia sobre el Futuro de Europa.

https://futureu.europa.eu/pages/information-material?locale=es 


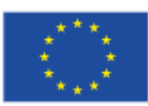

\section{Conferencia}

sobre el Futuro

de Europa

\section{Los paneles de ciudadanos europeos}

Los paneles de ciudadanos europeos son un elemento clave de la Conferencia sobre el Futuro de Europa, un ejercicio pionero en la democracia deliberativa en la Unión Europea.

Los paneles dan a los ciudadanos un papel más importante en la configuración de las futuras políticas y ambiciones de la Unión. Una muestra representativa de $\mathbf{8 0 0}$ ciudadanos se reunirá y debatirá sobre el futuro de Europa para formular recomendaciones políticas sobre los principales temas abordados en la plataforma digital multilingüe de la Conferencia.

Dichas recomendaciones se incorporarán a los debates generales de la Conferencia para su seguimiento.

\section{¿Quién formará parte de los paneles?}

- Cuatro paneles, cada uno de ellos compuesto por 200 ciudadanos de los veintisiete Estados miembros, seleccionados de modo aleatorio

- Representativos de la diversidad de la UE: origen geográfico, sexo, edad, situación socioeconómica y nivel educativo

- Un tercio de jóvenes (16-25) en cada panel

\section{¿Qué se debatirá?}

- Cuatro paneles que cubren temas diferentes ofrecen el espacio para un debate ascendente:

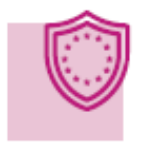

valores, derechos, estado de Derecho, democracia, seguridad

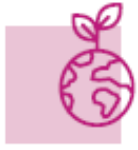
cambio climático, medio ambiente/salud

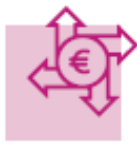

una economía más fuerte, justicia social, empleo/ educación, juventud, cultura, deportes/

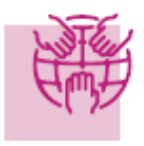
la UE en el mundo/ migración transformación digital

\section{¿Cuál será el resultado de los paneles?}

- Los paneles debatirán las aportaciones recogidas a través de la plataforma digital. Formularán una serie de recomendaciones para su seguimiento por parte de la Unión, y éstas se debatirán en el Pleno de la Conferencia.

- 80 representantes de cada panel de ciudadanos europeos, de los cuales al menos un tercio será menor de 25 años, participarán en el Pleno a fin de presentar los resultados de sus debates y de debatir con otros participantes.

- Se mantendrá informados a los participantes de cada panel sobre el seguimiento de sus recomendaciones. Al final del proceso se celebrará un acto dedicado a la información de retorno.

Fuente: Comisión Europea.

https://ec.europa.eu/commission/presscorner/detail/es/FS_21_3032 


\section{Ratificación del Acuerdo post-Brexit en el Parlamento Europeo}

En su sesión plenaria del 28 Abril, el Parlamento Europeo ratificó por abrumadora mayoría (660 votos a favor, 5 en contra y 32 abstenciones) el acuerdo post-Brexit acordado en la Nochebuena de 2020.

De esta forma, el Acuerdo de Comercio y Cooperación, que entró en vigor de forma provisional el 1 de enero de este año, quedaba listo para entrar en vigor de manera definitiva.

El debate previo a la votación, que se prolongó durante más de cuatro horas y media, se convirtió en la antesala del momento histórico que significaba la ratificación del primer acuerdo comercial con un antiguo Estado miembro de la UE.

El voto favorable, sin embargo, no ocultaba la desconfianza de Bruselas hacia el Gobierno británico, y en particular sobre la aplicación del protocolo del acuerdo sobre la provincia británica de Irlanda del Norte. Durante el debate, numerosos europarlamentarios reclamaron a la Comisión Europea que extremase la vigilancia para garantizar que Londres cumpla lo pactado y evitar que Irlanda del Norte se convierta en un «agujero de entrada» de productos hacia el mercado interior europeo.

Recordaban, sin duda, que el 3 de marzo, de forma unilateral e inesperada, el gobierno británico había anunciado que el 1 de abril no aplicaría controles aduaneros y fronterizos a las mercancías procedentes de Gran Bretaña con destino a la provincia británica de Irlanda del Norte, y que los aplazaba seis meses, hasta el 1 de octubre.

Tras la primera reacción de Bruselas, que decidió abrir un procedimiento de infracción contra el gobierno de Boris Johnson a mediados de Marzo, la Comisión y el Gobierno británico han mantenido un diálogo sobre esta eventual violación del protocolo específico para Irlanda del Norte contenido en el acuerdo de retirada.

«En los últimos días y semanas, hemos asistido a una dinámica nueva y constructiva, y seguiremos trabajando estrechamente con el Reino Unido para encontrar soluciones que respeten lo acordado. El siguiente paso es acordar mutuamente las vías de cumplimiento, con plazos e hitos concretos», señalaba la presidenta de la Comisión Von der Leyen ante el pleno del Parlamento Europeo.

«Coincido con ustedes en que este acuerdo sobre el papel sólo es tan bueno como su aplicación y cumplimiento en la práctica. Y comparto su preocupación por las acciones unilaterales del Reino Unido desde que el acuerdo entró en aplicación provisional. Evidentemente, hemos visto surgir una serie de problemas desde entonces», dijo Von der Leyen. La presidenta de la Comisión incidió en que «las decisiones unilaterales no llevan a ninguna parte» y pidió «centrarse en soluciones conjuntas», al tiempo que 
recordó que el acuerdo comercial contiene precisamente las herramientas «para garantizar el pleno cumplimiento de las obligaciones» que ambas partes acordaron al poner su firma en el acuerdo.

El negociador jefe de la UE para el Brexit, Michel Barnier, quien recibió un prolongado aplauso de despedida de los eurodiputados en reconocimiento a su trabajo, señaló que «todo el mundo debe asumir la responsabilidad de lo que se ha firmado» y también recordó las palabras de la diputada laborista asesinada en vísperas del referéndum de 2016, Jo Cox, quien afirmó entonces que la UE y el Reino Unido «tienen más en común que lo que les divide».

«El Brexit es un aviso y es un fracaso de la UE. Tenemos que aprender lecciones de ello como políticos en las instituciones y en las capitales. ¿Por qué un $52 \%$ de los británicos votó en contra de la UE?», se preguntó Barnier, apuntando a continuación a razones como «la tensión social y el enfado», por lo que recordó que el deber de los políticos es «escuchar y entender» a los ciudadanos.

Con la aplicación definitiva del Acuerdo, la Comisión deberá reforzar sus esfuerzos para prevenir el dumping social, fiscal y ambiental por parte del Reino Unido; la UE deberá garantizar también la competencia leal de los británicos en su acceso al mercado único de servicios financieros, condicionado al cumplimiento de una serie de compromisos sobre cooperación fiscal y lucha contra el blanqueo de capitales.

\section{BCE: inicio del debate sobre la retirada de estímulos}

El Banco Central Europeo afronta una encrucijada clave ante los síntomas de recuperación económica en la Eurozona.

Tras su enérgica respuesta contra la crisis del coronavirus, que le llevó a aprobar en Marzo de 2020 un Programa de Compra de Activos contra la Pandemia («Pandemic Emergency Purchase Programme», PEPP, por sus siglas en inglés) y que en Diciembre alcanzó la cifra de 1,85 billones de euros, se vislumbra ya el inicio del complejo debate sobre el ritmo al que se debería comenzar a retirar estos estímulos monetarios.

Las fricciones internas en el seno del Consejo de Gobierno del BCE (formado por los seis miembros de su Comité Ejecutivo y los 19 gobernadores de los Bancos Centrales de la Eurozona) no son un secreto, ni cuando se decidieron las sucesivas ampliaciones del programa en Junio y Diciembre de 2020, ni en estos momentos, cuando ya se escuchan opiniones de los más ortodoxos, los conocidos como «halcones», partidarios de la retirada de los estímulos.

Por el momento, el Consejo de Gobierno ha dejado claro que mantendrá las compras, al menos, hasta marzo de 2022 y que reinvertirá los vencimientos hasta 2023, pero a partir de estas decisiones todo son incógnitas. 
El enfrentamiento entre «palomas» $\mathrm{y}$ «halcones» es evidente, y en los últimos meses este debate se ha visto personalizado en las posiciones antagónicas del italiano Fabio Panetta, miembro del Comité Ejecutivo del BCE, quien alerta de los peligros de una retirada prematura de los estímulos que podría poner en peligro la recuperación, y la del holandés Klaas Knot, gobernador del Banco Central de los Países Bajos, quien ha destacado que la recuperación avanza más rápidamente de lo esperado y que el repunte de la inflación, que en Mayo alcanzó el 2\% en la Eurozona justificarían el no alargamiento innecesario de dichos estímulos.

El comportamiento de la inflación, aunque centrado en los precios de la energía, ha dado nuevos argumentos al sector más ortodoxo del BCE, y sitúa a la autoridad monetaria en una disyuntiva difícil, ya que deberá encontrar el complicado equilibrio que defina el momento y los ritmos para retirar las ayudas sin que estas decisiones comprometan el camino hacia la recuperación económica. En efecto, la palabra «tapering», que alude a la retirada gradual de estímulos, podría conducir a una subida de las primas de riesgo y, por tanto, de los costes de financiación de los Estados.

\section{Consejo Europeo de Oporto: reactivación de la Agenda Social}

El Consejo Europeo celebrado en Oporto en el mes de Mayo acordó la reactivación de la Agenda Social en la UE con el objetivo de frenar las desigualdades sociales mediante un plan de acción que despliegue políticas de empleo, igualdad de género y educación.

Aunque ya en otoño de 2017 la UE anunció una batería de medidas para imprimir un giro social a sus políticas después de años de austeridad, este «Pilar de los Derechos Sociales» ha tenido un alcance muy limitado desde entonces.

Y es por ello que, apenas cuatro años después de la cumbre de Gotemburgo que alumbró esta Agenda social y cuando, al igual que entonces, la UE parece vislumbrar la salida de esta nueva crisis a medida que el ritmo de la vacunación se acelera, los líderes de la Unión Europea se muestran preocupados y alerta sobre el riesgo de que la crisis económica se convierta en una grave crisis social.

Es así como la presidencia portuguesa se fijó el reto de impulsar el plan de acción propuesto previamente por la Comisión Europea para revitalizar el Pilar social de la UE, y que contiene diferentes iniciativas como la promoción de una vivienda asequible, la inversión en los sistemas de salud o la mejora de las capacidades laborales de los europeos.

Según afirmó la presidenta de la Comisión Europea, Ursula von der Leyen, «si en Gotemburgo se trató de principios, en Oporto se trata de acción. 
Se trata de convertir esos principios en un cambio positivo que sea tangible para millones de europeos».

El consenso del Consejo Europeo llegó tras superar los recelos procedentes de los países del norte, que reivindican sus competencias nacionales en políticas como el salario mínimo, y, también, la oposición de Polonia y Hungría, que trataron hasta el final de evitar cualquier referencia a la «igualdad de género».

En la declaración final, los Veintisiete abogaban por «profundizar en la implementación del Pilar Social», que los países consideran «un elemento fundamental de la recuperación», aunque «con el debido respeto por las respectivas competencias y los principios de subsidiariedad y proporcionalidad», afirmación que hacía referencia, sin duda, a la controvertida propuesta de un sueldo mínimo en las retribuciones de los trabajadores europeos.

«Estamos comprometidos en reducir desigualdades, defender salarios justos, combatir la exclusión social y la lucha contra la pobreza, asumiendo el objetivo de luchar contra la pobreza infantil, y abordar los riesgos de exclusión de grupos sociales especialmente vulnerables como los parados de larga duración, las personas mayores, las personas con discapacidades y las personas sin hogar», apuntaba el texto.

Los líderes de la UE también afirmaron que darán prioridad a los jóvenes, puesto que «se han visto muy perjudicados por la crisis de la covid-19, viendo obstaculizada su participación en el mercado laboral, así como sus planes de educación y formación». De hecho, los Veintisiete se comprometen a situar la «educación» y «las capacidades» en «el centro» de la acción política, puesto que las transiciones ecológica y digital darán nuevas oportunidades, pero también requerirán de nuevas destrezas laborales.

\section{Cuestiones generales de la actualidad económica}

\section{Suspensión del Pacto de Estabilidad y Crecimiento: debates sobre su activación y futuro diseño}

Los debates en torno a la suspensión del Pacto de Estabilidad y Crecimiento se han sucedido a lo largo del semestre. Se iniciaron ya a comienzos de Marzo, cuando la Comisión Europea propuso mantener su suspensión también en 2022, sobre la base de la publicación de sus previsiones económicas de invierno, que no situaban la recuperación económica a niveles pre-pandemia hasta la segunda mitad de 2022.

Y en Junio esta propuesta, defendida de forma oficiosa hasta entonces, fue presentada formalmente por tres comisarios, el vicepresidente econó- 
mico Valdis Dombrovskis, el comisario de Economía Paolo Gentiloni, y el comisario de Empleo y Derechos Sociales Nicolas Schmit.

Los tres insistieron en la necesidad de que los Estados miembros no retiren de forma prematura los estímulos económicos, ya que es preciso priorizar la recuperación económica a la consolidación fiscal para, como señalaron «no volver a caer en los errores de sacrificar la inversión pública y el gasto productivo», tal como se hizo en la crisis financiera de 2008.

Los comisarios plantearon, por tanto, mantener en suspenso las reglas fiscales contempladas en el PEC en 2022, prorrogando así la decisión adoptada en Marzo de 2020. Y, también, mostraron la predisposición de la Comisión a debatir una reforma de dichas reglas que rebaje la disciplina fiscal a los Estados antes de su reactivación en 2023.

Debe tenerse en cuenta que estas normas establecen como referencias básicas un umbral de deuda pública máximo del $60 \%$ sobre el PIB y que, en el marco del procedimiento de déficit excesivo, se exigen ajustes a partir de un agujero fiscal del $3 \%$ del PIB, cuando, actualmente, con la crisis de la pandemia ambos umbrales han sido superados en una gran mayoría de países. En España, la deuda pasó del 95,5\% en 2019 al 120\% en 2020, y el déficit escaló del $2,8 \%$ al $11 \%$ del PIB.

El comisario Dombrovskis señaló en rueda de prensa que «para conseguir la consolidación fiscal a medio plazo es importante tener un enfoque creíble» y que, para ello, «habrá que esperar a comprobar las secuelas permanentes que deja la crisis y la magnitud del impacto positivo de las ayudas europeas». Hasta entonces, afirmó, la medición de la salud fiscal de los países se hará con «criterios cualitativos y no cuantitativos».

Gentiloni, por su parte, pidió «prudencia fiscal» a los países con mayor endeudamiento público y les instó a tratar de reducir el aumento del gasto público estructural y a acometer inversiones que permitan potenciar el crecimiento, en particular en áreas verdes y digitales, aprovechando los fondos ligados a los Planes de recuperación. La clave, señaló, es «tener menos gasto corriente y más inversiones en investigación e infraestructuras» y reclamó, también, reformas para diseñar «más impuestos medioambientales» y para reforzar la «lucha contra la competencia fiscal».

Respecto a la eventual flexibilización de las reglas fiscales que podrían reactivarse eventualmente en 2023, ambos comisarios señalaron que no sería una decisión fácil dada la disparidad de posiciones e intereses de los Estados miembros. Destacaron que el debate debería abordarse ya en la segunda mitad de 2021 y que el objetivo final debería ser «simplificar las normas y reforzar los efectos contracíclicos» del PEC.

En todo caso, pronto se escucharon las posiciones contrapuestas en torno a este importante debate de política económica. Mientras el ministro austriaco de Finanzas, Gernot Blümel, se mostraba a finales de Junio 
«preocupado» por quienes cuestionan las reglas fiscales y aseguraba que «el objetivo común debe ser reducir la deuda», en el lado opuesto, el primer ministro italiano, Mario Draghi, reclamaba «estímulos adicionales», para regresar a la prudencia fiscal únicamente cuando se vuelva a los niveles de actividad precrisis, «cuando la recuperación sea autosostenida», señaló.

El debate será intenso, ya que las posturas difieren enormemente; por un lado, están los países llamados «halcones», liderados por Austria y Países Bajos, que defienden la austeridad en las finanzas públicas, y por otro, están los países del sur, encabezados por Francia, Italia y España, que reclaman una mayor flexibilidad y discrecionalidad en la aplicación de las reglas fiscales que se apliquen a partir de 2023.

La realidad muestra que, en cualquier caso, el límite del $60 \%$ de deuda sobre PIB es considerada una quimera con las cifras actuales de endeudamiento, por lo que, en opinión de muchos expertos el camino hacia unas cuentas públicas saneadas y sostenibles deberá definirse en base a una estrategia de reducción gradual de esta deuda con objetivos diferentes para cada país, aplicando una regla de gasto (una limitación del aumento del gasto público teniendo en cuenta la trayectoria del crecimiento).

\section{UE-EEUU: fin del conflicto arancelario por las ayudas a Airbus y Boeing}

La visita del nuevo presidente norteamericano Joe Biden a las instituciones de la UE en Bruselas a mediados de Junio se saldó con el esperado anuncio del fin de la «guerra comercial» entre los bloques provocada por la disputa de las ayudas a Airbus y Boeing.

Bruselas y Washington alcanzaron un acuerdo para suspender la imposición de aranceles mutuos y encontrar una solución duradera a una disputa de casi 20 años, y que estaba generando un daño económico de unos 11.500 millones de dólares (unos 9.500 millones de euros).

El pacto, que significa la suspensión durante cinco años de las tarifas impuestas a raíz del conflicto provocado por los subsidios a los grupos aeronáuticos Airbus y Boeing, mostraba, en palabras de la presidenta de la Comisión Europea Ursula Von der Leyen, «el nuevo espíritu de cooperación entre la UE y EEUU y la posibilidad de resolver los problemas para nuestro beneficio mutuo».

El ex presidente estadounidense Donald Trump había impuesto en 2019 aranceles por valor de 7.500 millones de dólares a las exportaciones europeas, una decisión contestada un año después por parte de la UE en forma de aranceles por valor de 4.000 millones de dólares a las exportaciones estadounidenses. En total, sumaban más de 11.500 millones de dólares en una represalia cruzada autorizada por la Organización Mundial del Comercio, y cuyo 
origen se remontaba al año 2004, cuando Estados Unidos presentó la primera queja ante la OMC por las ayudas de la UE a Airbus en forma de créditos con intereses muy inferiores a los de mercado para el desarrollo de sus nuevos aviones, lo que perjudicaban los intereses y la competitividad de su rival estadounidense Boeing. También la UE denunció posteriormente ante el organismo multilateral las ayudas en forma de incentivos fiscales recibidos por la propia Boeing.

La noticia de esta «paz comercial» fue recibida con satisfacción por gran parte del sector agrícola español, ya que los aranceles americanos afectaban a productos que les afectaba directamente, como el vino, el aceite o el queso.

Desde la Federación Española de Industrias de Alimentación y Bebidas (FIAB) señalaban que «desde su imposición en octubre de 2019, un total de 113 categorías de productos españoles, que representan el 53,1\% sobre el total de productos exportados a EEUU, se han visto gravados con aranceles del $25 \%$, causando un grave perjuicio en productos tan significativos como el aceite de oliva, el vino, el queso, la aceituna de mesa, la carne de porcino, los zumos, el procesado de pescados o las bebidas espirituosas».

\section{Fiscalidad: Información obligatoria de las grandes corporaciones sobre beneficios e impuestos «país por país»}

Cinco años después de que la Comisión Europea lanzara una propuesta en 2016, el Parlamento Europeo y el Consejo alcanzaron, a comienzos de Junio de este año, un importante acuerdo para la aprobación de una Directiva que obligará a las grandes multinacionales a detallar en sus cuentas información sobre su presencia en cada país, con una breve descripción de su actividad, el número de empleados, el volumen neto de negocios, los beneficios o pérdidas antes de impuestos, los tributos devengados y pagados al fisco y los beneficios acumulados.

Además de facilitar estos datos sobre su actividad e impuestos pagados en los países de la UE, también deberán facilitar el mismo detalle respecto a los Estados incluidos en la «lista negra» de la UE de paraísos fiscales (territorios que no cumplen los estándares mínimos de cooperación y transparencia fiscal), y respecto a los que lleven dos años o más en la «lista gris» (aquellos que tampoco cumplen, pero se han comprometido a enmendar su legislación).

Este detalle en las cuentas de las empresas será un elemento esencial en la transparencia de la actividad de las grandes corporaciones, y permitirá conocer con mayor claridad la ingeniera financiera y las prácticas de evasión fiscal.

La medida, que afectará a las grandes corporaciones, cuya facturación anual sea superior a 750 millones de euros durante más de dos ejercicios consecutivos (y a sus filiales, sin que importe su tamaño, si se considera 
que existen solo para ayudar a la matriz a eludir este requisito), salió adelante con algunas concesiones del Parlamento Europeo, como la aprobación de la cláusula de salvaguardia de cinco años a la que podrían acogerse las empresas (y dejar de reportar su actividad en un territorio) en el caso de que justificaran que esta información pudiera afectar a su competitividad.

La Directiva deberá ser revisada dentro de cuatro años, con las aspiraciones declaradas por el Parlamento de rebajar el umbral de facturación necesario para que una empresa deba presentar estos informes, ampliar el alcance geográfico de la información hasta tener que informar sobre la actividad en todo el mundo y limitar la cláusula de salvaguarda mencionada.

La aprobación definitiva de la Directiva requerirá, en todo caso, las votaciones finales en el pleno de la Eurocámara y en el Consejo que, previsiblemente, tendrán lugar después del verano.

Una vez aprobada la Directiva, la UE pondrá en marcha una iniciativa que es conocida como el 'Country by Country Report' y que llevaba cinco años sobre la mesa a partir de un proyecto de la OCDE aunque, de hecho, ya se aplica en una mayoría de Estados miembros, entre ellos, España,

\section{Competencia: investigaciones por prácticas monopolísticas de Facebook y Google}

Después de una investigación preliminar, los servicios de la Competencia del ejecutivo comunitario anunciaron el 4 de Junio el inicio de una investigación antimonopolio contra Facebook, al confirmar sus sospechas de que la tecnológica podría estar haciendo uso de los datos que recopila de los anunciantes que se publicitan en su red social, para competir con ellos en su servicio de anuncios clasificados Facebook Marketplace, una plataforma fundada en 2016 que ofrece a los usuarios de su red social la compraventa de artículos de forma gratuita.

Bruselas quiere examinar si estas prácticas perjudican la competencia en ese mercado, en la medida en que Facebook podría estar utilizando, en su caso, datos valiosos que manejan las empresas que se anuncian en la plataforma. Sospecha que, por ejemplo, Facebook podría estar recibiendo información precisa sobre las preferencias de los usuarios a partir de su interacción con los anuncios de sus competidores y que, con esa información, la corporación norteamericana podría estar adaptando Facebook Marketplace para imponerse a sus rivales.

«Casi 3.000 millones de personas usan Facebook mensualmente y casi siete millones de empresas se anuncian en él. Facebook recopila grandes cantidades de datos sobre las actividades de los usuarios de su red social y más allá, lo que le permite dirigirse a grupos de clientes concretos. Anali- 
zaremos detalladamente si estos datos le dan una ventaja competitiva indebida, en particular en el sector de los clasificados en línea, donde la gente compra y vende productos todos los días y donde Facebook también compite con empresas de las que recopila datos», afirmaba la Comisión en un comunicado.

Bruselas también examinará si la forma en la que Facebook Marketplace está integrado en la red social constituye «una forma de vinculación» que le da ventaja para llegar a los clientes e imponerse a la competencia.

Por su parte, el 22 de junio el ejecutivo comunitario hizo pública su decisión de iniciar otra investigación formal antimonopolio contra Google para analizar, en este caso, sus prácticas en el mercado de la publicidad online y determinar si ha favorecido sus propios servicios de tecnología de publicidad gráfica online en detrimento de sus competidores, anunciantes y editores.

Bruselas pretende valorar si, en efecto, Google está distorsionando la competencia al restringir el acceso de terceros a los datos de los usuarios en sitios web y aplicaciones con fines publicitarios, mientras reserva dichos datos para su propio uso.

La comisaria responsable de la competencia, Margrethe Vestager, señaló que «los servicios de publicidad online son la base de cómo Google y los editores monetizan sus servicios en línea. Google recopila datos para utilizarlos con fines publicitarios específicos, vende espacios publicitarios y también actúa como intermediario, por lo que está presente en casi todos los niveles de la cadena de suministro de la publicidad online».

El Ejecutivo comunitario recordaba en su comunicado que muchos editores confían en este tipo de publicidad gráfica online para financiar contenidos y publicaciones que ofrecen de forma gratuita en internet. Y estimaba que, en 2019, el volumen de gasto en la publicidad online en la UE alcanzó los 20.000 millones de euros.

Según Vestager, la igualdad de condiciones es fundamental para todos los integrantes de la cadena de suministro. «La competencia leal es importante, tanto para que los anunciantes lleguen a los consumidores en los sitios de los editores, como para que los editores vendan su espacio a los anunciantes para generar ingresos y financiación para el contenido», señaló.

Bruselas examinará, entre otros temas, las condiciones que impone la tecnológica para la compra de espacios publicitarios en You-tube o la obligación de usar su servicio de gestión de los anuncios. Y, también, las restricciones que impone a terceros para acceder a datos sobre la identidad y el comportamiento de los usuarios que sí están disponibles para los servicios de intermediación publicitaria del gigante tecnológico.

Google, por su parte, defendió sus servicios y señaló que «miles de empresas europeas utilizan nuestros productos publicitarios porque son com- 
petitivos y eficaces» y mostró el compromiso de la empresa en «seguir colaborando de forma constructiva con la Comisión para demostrar las ventajas de nuestros productos».

\section{Investigación sobre la compra de Air Europa por parte del grupo IAG}

La Comisión Europea decidió a finales de Junio iniciar una investigación sobre la adquisición de Air Europa por parte del grupo IAG, la matriz de Iberia, tras negar su autorización al proyecto de fusión, puesto que según el ejecutivo comunitario esta operación podría reducir la competencia en los mercados del transporte aéreo nacional e internacional.

Bruselas planteaba reservas en dos temas. Por una parte, teme que la propuesta planteada por IAG suponga «reducir significativamente» la competencia en 70 rutas nacionales o que tienen como origen o destino una ciudad española, en las que ambas compañías ahora compiten. «En varias rutas, IAG y Air Europa son las dos únicas compañías aéreas que las explotan», destacaba el comunicado. Y a escala internacional, la Comisión también se mostraba preocupada por varias rutas entre Madrid y los Estados Unidos y América Latina.

Y en segundo lugar, Bruselas teme el efecto que pueda tener la operación en otras compañías aéreas que dependen de la red nacional de corta distancia de Air Europa para sus operaciones en el aeropuerto de Madrid y en otros aeropuertos de la Unión Europea, ya que sin los vuelos de enlace que ahora ofrece Air Europa, muchas compañías podrían decidir suspender sus vuelos internacionales ante la competencia de IAG, lo cual podría resultar, finalmente, en una subida de tarifas aéreas para los pasajeros.

La Comisión valoraba también que ni siquiera las compañías de bajo coste lograrían ejercer una competencia suficiente en varias rutas con altas cuotas de mercado conseguidas por la nueva entidad.

«IAG, que opera con Iberia y Vueling, entre otros, y Air Europa son las principales compañías aéreas de España. También son proveedoras fundamentales de conexiones entre España, el resto de Europa y América Latina. Examinaremos cuidadosamente si la operación propuesta afectaría negativamente a la competencia en las rutas nacionales, de corta distancia y de larga distancia con origen y destino en España, lo que podría dar lugar a precios más elevados y a una menor calidad para los viajeros», sostuvo la comisaria de Competencia, Margrethe Vestager, en un comunicado.

En este primer análisis, Bruselas señalaba que serán necesarias concesiones y compromisos por parte de IAG para que la operación, notificada en mayo, siga adelante. 


\section{Reforma de la Política Agrícola Común}

El 28 de Junio los países de la Unión Europea dieron luz verde al acuerdo sobre la reforma de Política Agrícola Común, PAC, para el periodo 2023-2027, con el objetivo de afrontar los retos ambientales y climáticos a través de una producción más sostenible.

Durante el debate público del Consejo de ministros de Agricultura celebrado en Luxemburgo se analizó el acuerdo logrado previamente entre los representantes de las instituciones europeas (Consejo y Parlamento con la mediación de la Comisión Europea), que establecía una serie de medidas destinadas a conseguir que los agricultores adopten prácticas agrícolas «más ecológicas» y que incluía una serie de normas sobre condicionalidad que los agricultores deberán cumplir para recibir el apoyo de la PAC, incluida la conservación de suelos ricos en carbono mediante la protección de humedales y turberas, y unas cuotas mínimas de tierra cultivable para proteger la biodiversidad.

La reforma incluye la novedad de los «ecoesquemas» con los que, en efecto, se pretende apoyar e incentivar a los agricultores para que desarrollen e implementen prácticas agrícolas respetuosas con el medio ambiente. El objetivo de la reforma no es otro que alinear la política agrícola al Pacto Verde europeo para impulsar una producción y un consumo más sostenibles.

«Es la mayor reforma de la PAC desde los 90. Teníamos la ambición de lograr una agricultura más sostenible, justa, inclusiva y que nos proporcione autonomía estratégica y lo hemos logrado», señaló la ministra de Agricultura de Portugal, Maria do Céu Antunes, cuyo país ha presidido la UE este semestre.

Las instituciones deberán ahora concluir los detalles técnicos de la reforma propuesta, tras lo cual será aprobada formalmente por el Parlamento Europeo y el Consejo, para poder ser aplicada en 2023.

Con todo, las organizaciones agrarias españolas mostraron su recelo por el destino de los fondos de la PAC a las políticas verdes, que incluirán, efectivamente, nuevas exigencias medioambientales que supondrán, eventualmente, mayores costes de producción y la pérdida de competitividad frente a las importaciones extracomunitarias, que se benefician de menores estándares y exigencias laborales, medioambientales y de seguridad alimentaria.

El sector teme que la reforma pudiera derivar en pérdidas de rentabilidad, cierres de pequeñas y medianas explotaciones y una menor autosuficiencia alimentaria para la Unión Europea. 


\section{Derechos de autor}

Los derechos de autor (para la distribución, comunicación pública, reproducción e inclusión en bases de datos de indexación y repositorios institucionales) de esta publicación (Cuadernos Europeos de Deusto, CED) pertenecen a la editorial Universidad de Deusto. El acceso al contenido digital de cualquier número de Cuadernos Europeos de Deusto es gratuito inmediatamente después de su publicación. Los trabajos podrán leerse, descargarse, copiar y difundir en cualquier medio sin fines comerciales y según lo previsto por la ley; sin la previa autorización de la Editorial (Universidad de Deusto) o el autor. Así mismo, los trabajos editados en CED pueden ser publicados con posterioridad en otros medios o revistas, siempre que el autor indique con claridad y en la primera nota a pie de página que el trabajo se publicó por primera vez en CED, con indicación del número, año, páginas y DOI (si procede). Cualquier otro uso de su contenido en cualquier medio o formato, ahora conocido o desarrollado en el futuro, requiere el permiso previo por escrito del titular de los derechos de autor.

\section{Copyright}

Copyright (for distribution, public communication, reproduction and inclusion in indexation databases and institutional repositories) of this publication (Cuadernos Europeos de Deusto, CED) belongs to the publisher University of Deusto. Access to the digital content of any Issue of Cuadernos Europeos de Deusto is free upon its publication. The content can be read, downloaded, copied, and distributed freely in any medium only for non-commercial purposes and in accordance with any applicable copyright legislation, without prior permission from the copyright holder (University of Deusto) or the author. Thus, the content of CED can be subsequently published in other media or journals, as long as the author clearly indicates in the first footnote that the work was published in CED for the first time, indicating the Issue number, year, pages, and DOI (if applicable). Any other use of its content in any medium or format, now known or developed in the future, requires prior written permission of the copyright holder. 